\title{
La cuantificación demográfica y epidemiológica en el higienismo balear, 1850-1930
}

\author{
Joana-Maria Pujadas-Mora (*) \\ $\left.{ }^{*}\right) \quad$ Centre d'Estudis Demogràfics. Universitat Autònoma de Barcelona. \\ jpujades@ced.uab.es
}

Dynamis

[0211-9536] 2012; $32(1): 165-184$
Fecha de recepción: 29 de junio de 2011

Fecha de aceptación: 17 de octubre de 2011

SUMARIO: 1.- Introducción. 2.-El afán y el método cuantificador. 3.-Los elementos configuradores del estado de salud de la población. 3.1.-El crecimiento y la distribución por sexos de la población. 3.2. - La estacionalidad de la mortalidad en la búsqueda de la relación entre clima y salud. 3.3.-El perfil demográfico y epidemiológico de la población. 4.-Waloraciones finales.

RESUMEN: El desarrollo de la Medicina Social potenció el uso de la cuantificación como herramienta para la valoración del estado de salud de las poblaciones. En Mallorca, higienistas como los médicos Enric Fajarnés, Bernat Riera, Antoni Mayol y Emili Darder y el ingeniero de caminos Eusebi Estada buscaron conocer el estado de salud a través del crecimiento de la población, el perfil demográfico y epidemiológico de la mortalidad y su relación con el clima. Sus cálculos mostraron el buen estado de salud de la población balear respecto a la española, mientras no era tan satisfactorio en un contexto internacional. Estos resultados se explicaban por la benevolencia del clima insular, lo que justificaba la reforma sanitaria.

PALABRAS CLAVE: Cuantificación, estado de salud, crecimiento de la población, perfil demográfico y epidemiológico, siglos XIX y XX.

KEY WORDS: Quantification, health status, population growth, demographic and epidemiological profile, 19th and 20th centuries.

\section{Introducción $(*)$}

El uso de la cuantificación demográfica y/o epidemiológica como herramienta para la valoración del estado de salud de la población se intensi-

(*) La investigación para este artículo se desarrolló en el marco del proyecto de investigación «La acción de la Administración y de la Iglesia en el proceso de dotación de servicios públicos en España (1845-1930). Un análisis regional» bajo la dirección de la Dra. Isabel Moll, como parte de mi tesis doctoral, Evolució de la mortalitat infantil i juvenil a la ciutat de Palma (Mallorca, 18381960) presentada en la Universitat de les Illes Balears en julio de 2009. 
ficó a finales del siglo XIX, como parte del desarrollo de la denominada Medicina Social ${ }^{1}$. De esta manera se pretendía objetivar el estado de salud de la población a través, inicialmente, de la estimación numérica de la mortalidad. De su lectura y/o interpretación se producía la visibilización del problema sanitario y se desprendía su posible solución en forma de actuación normalmente pública ${ }^{2}$ legitimada por los propósitos del programa higienista. Este programa en su versión urbana se fijó en el derribo de las murallas, la canalización del agua y del alcantarillado, la higiene de las viviendas para evitar el hacinamiento y la mala ventilación, la construcción de ensanches, etc. ${ }^{3 .}$

El afán cuantificador corresponde al desarrollo epistemológico en la búsqueda de la objetividad científica con la creación de un conocimiento impersonal de los problemas o fenómenos que interesa analizar ${ }^{4}$. Se trata de un afán que en España se vio favorecido por el desarrollo del Estado de Derecho. La institucionalización de este nuevo modelo de organización política produjo el refuerzo de la Administración pública desde la racionalización de su estructura a partir del conocimiento de la composición de su población o de sus fuentes de riqueza ${ }^{5}$, lo cual exigía crear un aparato de recogida y tratamiento de información a nivel estatal para cuantificar esta información. Con el Real Decreto de 3 de noviembre de 1856 se creó la Comisión de Estadística General del Reino que permitió la confección del primer censo de población que inicia la época estadística en $1857^{6}$.

1. Rodríguez Ocaña, Esteban. La constitución de la Medicina Social como disciplina en España, 1884-1923. Madrid: Ministerio de Sanidad y Consumo; 1987; Rodríguez Ocaña, Esteban. Medicine as a social political science. Hygiea. 2007; 7: 37-52.

2. Armstrong, David. The invention of infant mortality. Sociology of Health \& IIIness. 1986; 8 (3): 211-232; Rusnock, Andrea. Quantifying infant mortality in England and France 1750-1800. In: Jorland, Gérard; Opinel, Annick; Weisz, George, eds. Body counts-medical quantification in historical and sociological perspectives. Montreal: McGill-Queen's University Press. 2005, p. 65-86.

3. Bujosa Homar, Francesc. Los sorprendentes índices sanitarios en la Mallorca de finales de siglo. Dynamis. 1998; 18: 233-250. Rodríguez Ocaña, Esteban. Comodidad, ornamentación, higiene. Modernización urbana e higienismo en la España del siglo XIX. In: Salud pública en España: ciencia, profesión y política, siglos XVIII-XX. Granada: Editorial Universidad de Granada, 2005.

4. Porter, Theodore. Trust in numbers. The pursuit of objectivity in science and public life. Princeton: Princeton University Press; 1995.

5. Porter, n. 4; Woolf, Stuart. Statistics and the modern state. Comparative Studies in Society and History. 1989; 31 (3): 588-603.

6. Muro, José Ignacio; Urteaga, Luis; Nadal, Francesc. Geografía, estadística y catastro en España, 1856-1870. Barcelona: Ediciones del Serbal; 1996. 
Desde esa fecha, con más o menos periodicidad y con la refundación de su institución regente, se fueron alzando censos de población para el conjunto del Estado hasta el año 2001, cuando pasó a ser un padrón continuo. Por otra parte la publicación de la estadística ${ }^{7}$ de flujos demográficos se inició en España en 1863 con la Memoria sobre el movimiento de la población en España en los años 1858, 1859 y 1861 por parte de la Junta General de Estadística del Reino ${ }^{8}$. Esta estadística continúa llevándose a cabo año a año bajo el nombre de Movimiento natural de la población. Su confección se realizó en un primer momento a partir de los registros sacramentales y luego del Registro Civil. Este último fue instaurado definitivamente en 1871, después de toda una serie de intentos fallidos por parte de la Administración liberal ${ }^{9}$.

Las estadísticas regulares de carácter epidemiológico se iniciaron en 1879 con el Boletín Mensual de Estadística Demográfica Sanitaria de la Península e Islas Adyacentes, seguido por el Boletín de Sanidad entre 1888 y $1896^{10}$, a pesar de que en el Movimiento natural de la población también se incluyeran de manera paulatina datos relativos a la distribución de causas de muerte por edades, sexos, etc. La creación de este tipo de estadísticas fue una de las aspiraciones de la medicina del siglo XIX en España o realizaciones en otros Estados europeos ${ }^{11}$.

7. La palabra estadística tiene varios significados que podrán ser utilizados a lo largo del texto. Uno de ellos es el conjunto de datos cuantitativos de la población. El estudio de estos datos también puede denominarse estadística. También responderá a la definición de «Rama de la matemática que utiliza grandes conjuntos de datos numéricos para obtener inferencias basadas en el cálculo de probabilidades» como reza para los tres significados el Diccionario de la Lengua Española de la Real Academia Española (consultado el 17 de enero de 2011). Los términos a los cuales recurriremos más habitualmente son los relativos al almanaque de datos y al de disciplina científica.

8. Cusidó Vallverdú, Teresa Antònia. Origen i història del moviment natural de la població a Espanya, 1850-1975. Universitat Autònoma de Barcelona; 2006 (memoria de doctorado).

9. Muro; Urteaga; Nadal, n. 6; Valero Escandell, José Ramón. La implantación del registro civil en España (problemas de utilización en estudios demográficos). Anales de la Universidad de Alicante. 1986; 5: 87-99.

10. Rodríguez Ocaña, Esteban; Bernabeu Mestre, Josep. Physicians and statisticians: two ways of creating demographic health statistics in Spain, 1841-1936. Continuity and Change. 1997 (12): 247-264.

11. Bernabeu Mestre, Josep. Estadística y salud pública: el argumento del método numérico. Gaceta Sanitaria. 2007; 21 (5): 416-417; Cassedy, James H. American medicine and statistical thinking, 1800-1860. Cambridge: Harvard University Press; 1984; Jorland; Opinel; Weisz, n. 2; Magnello, Eileen; Hardy, Anne, eds. The road to medical statistics. Amsterdam: Rodopi; 2002; Matthews, J. Rosser. Quantification and the quest for medical certainty. Princeton: Princeton 
El desarrollo del cálculo numérico, a su vez, fue producto de la consolidación de disciplinas como la Demografía o la Epidemiología junto a la Estadística ${ }^{12}$. Un desarrollo disciplinar pionero en Inglaterra y Francia que se siguió de cerca, aunque con un cierto retraso, en España. Por otra parte su consolidación hay que entenderla, en parte, como consecuencia del desarrollo de la Administración Pública ${ }^{13}$.

El objetivo del presente estudio es analizar la cuantificación aplicada por los higienistas mallorquines en la valoración del estado de salud de la población balear y su lectura a nivel local. Estos higienistas en particular fueron un grupo de profesionales de la medicina y de la ingeniería que formaron parte del movimiento reformador en aras de la Salud Pública canalizado a través de instituciones como la Real Academia de Medicina y Cirugía, el Colegio Médico-Farmacéutico o consistorios municipales, participando en estos tanto como políticos o como asesores. Trataremos las publicaciones de los médicos Fernando Weyler (1808-1879), Antoni Mayol Vidal, Bernat Riera Alemany (1874-1926) y Emili Darder Cànaves (1895-1937) junto a las del ingeniero de caminos Eusebi Estada (1843-1934).

La objetivación de dicho estado de salud mediante el uso de datos estadísticos condujo a la utilización de los compendios que el propio Estado producía (estadística vitales, sanitarias y censos) y a la creación de unos propios y locales dada la inmediatez necesaria en los aspectos de salud. Las cifras de mortalidad junto a la búsqueda de la relación entre clima y salud y la construcción del perfil epidemiológico a través de las causas de defunción, básicamente de naturaleza infecciosa, constituyeron los elementos que posibilitaron la presentación del estado de salud.

University Press; 1995; Rodríguez Ocaña, Esteban; Bernabeu Mestre, Josep. El legítimo criterio aritmético. Los métodos cuantitativos en la Salud Pública española, 1800-1939. In: Emilio Sánchez-Cantalejo Ramírez, ed. Quinto encuentro Marcelino Pascua. La epidemiología y la estadística. Granada; Escuela Andaluza de Salud Pública; 1996, p. 9-33.

12. La Berge, Ann F. Mission and method: The early nineteenth-century public health movement. Cambridge: Cambridge University Press; 1992; Porter, Dorothy. Health, civilization, and the state: a history of public health from ancient to modern times. London: Routledge; 1999; Schweber, Libby. Disciplining statistics. Demography and vital statistics in France and England, 1830-1885. Durham: Duke University Press; 2006.

13. Porter, n. 4. 


\section{El afán y el método cuantificador}

La cuantificación médica se desarrolló mediante la creación del objeto de estudio a partir de la recogida de datos demográficos y epidemiológicos, ya fuese desde fuentes primarias (trabajo de campo o documentos manuscritos, como el registro parroquial o el Registro Civil) o secundarias (como el Movimiento natural de la población, como la fuente más representativa del periodo de estudio) y su explotación a partir de una estricta metodología estadística.

La disponibilidad de fuentes estadísticas oficiales de carácter estatal en la segunda mitad del siglo XIX permitió la proliferación de los estudios sanitarios apoyados en datos demográficos y epidemiológicos en el contexto español. Estas estadísticas se vieron reforzadas por iniciativas locales como los estadillos mensuales de nacimientos y defunciones de la ciudad de Palma publicados en la Revista Balear de Ciencias Médicas ${ }^{14}$ del Colegio Médico-Farmacéutico ${ }^{15}$. Formaron una sección fija en dicha revista, desde su creación en 1885, con el nombre de Demografía Médica ${ }^{16}$. Su primera finalidad era la de servir de base para la elaboración de un topografía médica de la ciudad, la cual nunca llegó a materializarse ${ }^{17}$.

En esa sección se reportaron los nacimientos clasificados por sexo y legitimidad, las defunciones agrupadas según la edad de defunción y una distribución etiológica de las causas de muerte. Éstas fueron clasificadas en cuatro grupos: Enfermedades infecciosas, Otras enfermedades frecuentes, Otras enfermedades y Muerte violenta siguiendo la clasificación utilizada en el Boletín Mensual de Estadística Demográfico Sanitaria que recogía las recomendaciones del Congreso Internacional de Estadística celebrado en Budapest en $1876^{18}$ para el mes de enero de 1885 (que coincide con el

14. El órgano de difusión del Colegio Médico-Farmacéutico de Palma se inició en 1885 con periodicidad quincenal y el nombre de Revista Balear de Medicina, Farmacia y Veterinaria, cambiado desde enero de 1888 por Revista Balear de Ciencias Médicas. Oliver Capó, Gabriel. Análisis histórico de la Revista Balear de Ciencias Médicas (1885-1912). Universidad de Zaragoza; 1996.

15. Para más información ver: Tomás Monserrat, José. Medicina y sociedad: el colegio de médicos de Baleares 1882-1982. Palma: Colegio oficial de médicos de Baleares; 1985.

16. Este tipo de publicaciones también fueron desarrolladas en Barcelona por los médicos Josep Nin y Lluís Comenge como presenta: Rodríguez Ocaña, Esteban. La labor estadística de Luis Comenge (1854-1916) en el Instituto de Higiene Urbana. Dynamis. 1986; 5-6: 279-306.

17. Oliver Capó, n. 14.

18. Rodríguez Ocaña; Bernabeu Mestre, n. 10. 
primer número de la revista). A partir del mes de febrero del mismo año y hasta principios del siglo XX se utilizó la clasificación recomendada por la Real Orden de 5 de enero de 1885 por la cual las enfermedades se dividían en: Enfermedades infecciosas y contagiosas, Otras enfermedades y Muerte violenta. Aunque con la publicación del Boletín de Sanidad en 1888 se introdujo una nueva clasificación, la revista siguió la de 1885 hasta el año 1900, cuando se adoptó la clasificación de Bertillon como también ocurrió en el Movimiento Natural de la Población.

Junto a estos estadillos mensuales cabe destacar las Notas demográficas. Movimiento de población en Baleares publicadas en la revista del Colegio Médico-Farmacéutico o como folletos con el título de Anuarios elaboradas por el médico Enric Fajarnés Tur $(1858-1934)^{19}$. Estas notas siguieron la misma estructura gráfica que los estadillos mensuales de nacimientos y defunciones de Palma que aquella revista publicaba regularmente, aunque éstos cubrieron toda la geografía balear por partidos judiciales, al tiempo que proporcionaron recuentos de matrimonios. Sin embargo las defunciones no se presentaron distribuidas por edad. En total publicó de forma separada 12 notas demográficas, a lo largo de la segunda mitad de 1888 y primera de 1889 , de forma que el año de referencia de ocurrencia de los acontecimientos demográficos fuera 1888 .

Esas notas fueron unificadas posteriormente por su creador, el médico Fajarnés, en su Demografía Médica de Baleares - año 1888-, y las desmembró en parte para la Demografía sanitaria de Menorca, donde desarrolló de manera relevante la parte dedicada al matrimonio. Esta última publicación, junto a la de Demografía de las Islas Baleares durante el quinquenio de 1886-1990, publicada en 1894, y una versión muy similar publicada en 1901 con el nombre de Estudio Demográfico de Baleares, dejaron de ser almanaques estadísticos para pasar a ser estudios más elaborados, tanto desde una perspectiva metodológica como analítica.

Con semejante naturaleza, los Anuarios demográficos del mismo autor, reúnen los fenómenos demográficos del nacimiento, defunción y matrimonio paro los años 1896 y 1897. Estas estadísticas estaban destinadas a constituir

19. Para una aproximación detenida a la figura de este médico ibicenco puede verse: PujadasMora, Joana-Maria. La producció científica d'Enric Fajarnés i Tur (1858-1934). In: Prats Garcia, Ernest; Pujadas-Mora, Joana-Maria, eds. Enric Fajarnés i Tur (1858-1934), entre la història i la demografia. Palma: Conselleria d'Economia, Hisenda i Innovació. Govern de les Illes Balears; 2008, p. 35-78. 
el material de base de los capítulos de Demografía dinámica, estática y retrospectiva pertenecientes a una topografía médica para la ciudad de Palma que había sido propuesta por el autor en la sesión de la Real Academia de Medicina y Cirugía del 20 de febrero de 1896. Desgraciadamente, y como ya se ha señalado, esta topografía nunca se llevó a cabo.

Otra fuente local a señalar es el Boletín de Estadística Municipal de Palma con apartados referentes a Sanidad y a Beneficencia. A diferencia de la anterior, su creación se dio en las primeras décadas del siglo XX fue consecuencia del desarrollo de la administración pública local, que no de la iniciativa médica.

Los métodos de cálculo demográfico y epidemiológico aplicados a estos materiales estadísticos para la valoración del estado de la salud de la población balear corresponden a la estadística descriptiva. Solo se utilizaron proporciones, razones y tasas relativas básicamente al acontecimiento de la defunción y sus causas. También se utilizaron números absolutos presentados en forma de tablas de contingencia para mostrar la distribución del acontecimiento demográfico por meses de ocurrencia, sexo, estado civil, etc., tal como se explicaba para las «notas» y «anuarios» del médico Fajarnés. Con las proporciones de nacimientos, defunciones y matrimonios se buscaba calcular su intensidad, aunque sensu stricto ésta sólo se puede calcular mediante tasas. Estas proporciones se expresaron por sexo, estado civil y mes de ocurrencia en el mejor de los casos. Por edades se midieron las defunciones y los matrimonios; más éstos últimos que las primeras. Los nacimientos se analizaron en función de la legitimidad. Estas medidas de frecuencia pretendían establecer la mayor o menor probabilidad de ocurrencia de un determinado fenómeno demográfico, sobre todo el de la defunción, en función de determinados factores como la edad, estado civil, etc. De aquí la utilización de sus cruzamientos que se presentaron en forma de tablas.

A su vez se calcularon tasas brutas de mortalidad. Las tasas ponen en relación un flujo con un stock demográfico determinado, lo que permite calcular la frecuencia relativa con la que ocurre un acontecimiento demográfico en relación a la población media en un determinado periodo de tiempo. De forma más concreta se calculó un cociente específico referido a las muertes fetales o mortinatalidad. Este cálculo se realizó por medio de la proporción de nacidos muertos sobre el total de nacidos vivos. Los nacidos muertos agrupaban a los nacidos muertos propiamente dichos, a los muertos al nacer y a los muertos dentro de las primeras veinticuatro horas. 
Por lo tanto, eran considerados nacidos vivos todos los que tuvieran figura humana y hubieron sobrevivido veinticuatro horas fuera del seno materno (artículo 30 del Código Civil de 1889). Un cociente que obviamente adolecía de sobreestimación en el numerador y de subestimación en el denominador, el cual fue utilizado por Fajarnés Tur (1901) en su publicación La mortinatalidad en Palma de Mallorca durante un cuarto de siglo, 1872-1896. Aun así el propio autor manifestaba la problemática que representaba la no distinción por viabilidad de los nacidos muertos:

«Ningún punto de la demografía médica española ofrece más dificultades para el estudio que el relativo a los nacidos muertos. Entre estos, hay que distinguir los abortos, los nacidos vivos, y los fallecimientos durante las primeras veinticuatro horas del nacimiento; pero esta clasificación no puede establecerse, porque los datos aparecen englobados (como aparecen en la publicación del Movimiento Natural de la Población) y, por consiguiente, es imposible determinar la mortalidad intra-uterina y la proporcional según los meses de concepción y es imposible distinguir los verdaderos y los falsos nacidos muertos» ${ }^{20}$.

La estimación de la prevalencia de las enfermedades se realizó a partir de una aproximación a los datos de defunciones y no al número de individuos que padecían una determinada enfermedad, dado que las estadísticas demográfico sanitarias al uso no incluían enfermos sino únicamente defunciones. De esta manera se calculó un cociente a partir del número de defunciones por causa y la población total existente en un momento dado. Este cómputo fue más propio de estudios cronológicamente cercanos a una fecha censal dado el uso del número de habitantes como denominador del cociente. Por esto también se pudo calcular este cociente introduciendo el factor edad, obteniendo con ello las tasas específicas de mortalidad ${ }^{21}$.

Con el mismo fin se empleó la proporción de una causa o grupo de causas sobre el total de las defunciones, teniendo en cuenta el sexo con el fin de calcular la incidencia separada para mujeres y hombres. Fajarnés entendía que era una técnica indirecta, pero la empleaba ante la ausencia

20. Fajarnés Tur, Enric. La morti-natalidad en Palma de Mallorca durante un cuarto de siglo, 18721896. Palma: Tipografía de las hijas de Juan Colomar; 1900, p. 12.

21. Este tipo de cocientes fueron utilizados en el artículo de Fajarnés Tur, Enric. Epidemia de sarampión en Palma durante el año 1887. Palma: Imprenta Juan Colomar y Salas; 1888. 
de «los datos estadísticos (...) de invasiones.» ${ }^{22}$. Únicamente en el caso de la población militar se recogían propiamente los datos de morbilidad, lo que permitió relacionar las invasiones y las defunciones por sarampión en un determinado periodo ${ }^{23}$.

También se utilizaron medidas de tendencia central como la media. De ahí que se calculara la media diaria, mensual y por estación del número de defunciones. Al mismo tiempo se midió el crecimiento de la población utilizando la diferencia absoluta entre dos recuentos de población, distribuida homogéneamente entre los años que separaban los recuentos. Así se pudo establecer la media de habitantes que había disminuido o aumentado la población anualmente. Esta posibilitaba el cálculo del número de años necesarios para su duplicación, tal como Malthus popularizó en su Ensayo sobre el principio de la población y que Fajarnés refirió en sus trabajos ${ }^{24}$.

Estas medidas corresponden casi exclusivamente a la estadística descriptiva, sin atender a cálculos como las tablas de vida en aras de calcular la esperanza de vida propios del análisis demográfico ${ }^{25}$, o cálculos estadísticos como las correlaciones o la utilización de la teoría de la probabilidad que se estaban creando o usando en Inglaterra o Francia ${ }^{26}$. La sofisticación del cálculo estadístico en las ciencias de la salud en España se retrasó en comparación a otros países europeos produciéndose un importante involución con la Guerra Civil (1936-1939) ${ }^{27}$.

\section{Los elementos configuradores del estado de salud de la población}

El estado de salud de la población se identificó, en primera instancia, con el signo de crecimiento en el número de habitantes, para luego determinar sus cifras de mortalidad, habitualmente complementadas con la relación de sus causas más frecuentes, que se consideraban como las enfermedades dominantes. Aquellas, por ejemplo las infecciosas, sobre las que la bacteriología proporcionaba medios seguros de profilaxis, aparecían como

\footnotetext{
22. Fajarnés Tur, n. 21, p. 6.

23. Fajarnés Tur, n. 21.

24. Fajarnés Tur, Enric. Acrecentamiento de la población de Baleares en un siglo. Revista Balear de Ciencias Médicas. 1902; 21: 1-12; 35-42.

25. Rodríguez Ocaña; Bernabeu Mestre, n. 11.

26. Porter, n. 4.

27. Rodríguez Ocaña; Bernabeu Mestre, n. 11.
} 
evitables ${ }^{28}$, lo que no quita para que se continuara postulando una relación entre clima y salud, propia de la mentalidad ambientalista. De hecho, sirvió de justificación de la buena salud de los habitantes de Baleares, frente a lugares de clima más severo. Elementos que se presentaron en la producción científica de los higienistas baleares como la de los médicos Fernando Weyler, Enric Fajarnés Tur de quien se ha informado de manera reiterada, Antoni Mayol Vidal, Bernat Riera Alemany, Emili Darder Cànoves o la del ingeniero de caminos, Eusebi Estada (1843-1934) como explicaremos seguidamente. Estos higienistas nos ofrecen la lectura local y coetánea del estado sanitario balear, junto a la comparación con la situación sanitaria del resto de España y de algunos países europeos. Las cifras de mortalidad que manejaron estos autores muestran que las condiciones de las Baleares eran mejores que las del resto de España, como la historiografía actual ha probado con métodos estadísticos más precisos ${ }^{29}$. Aun así éstas fueron interpretadas en clave derrotista como parte del discurso de reforma sanitaria que propugnaba el higienismo. Un discurso que llevaba implícito la creación y modernización de infraestructuras urbanas. Pero a su vez esta lectura negativa fue posible gracias a comparaciones asimétricas, por método y por recorrido histórico.

\subsection{El crecimiento y la distribución por sexos de la población}

La vigorosidad de la población, como sinónimo de buena salud, se midió por su crecimiento. Es decir, un crecimiento natural positivo identificaba la buena salud de los habitantes. Este tipo de cálculos era herencia de las tesis malthusianas en relación a las progresiones y el desequilibrio entre población y recursos. Por esto se intentó estimar el tiempo necesario para la

28. Porras Gallo, María Isabel. La lucha contra las enfermedades "evitables" en España y la pandemia de la gripe de 1918-19. Dynamis, 1994; 14: 159-184.

29. Para una visión más detenida del tema ver entre otros: Lujosa Homar, n. 3; Bujosa Homar, Francesc; Moll Blanes, Isabel; Sureda García, Bernat. La avanzada transición demográfica en Mallorca: el caso de la mortalidad infantil. Boletín de la Asociación de Demografía Histórica. 2000; 28 (2): 125-146; Reher, David-Sven. El declive de la mortalidad en España, 1860-1930. Madrid: Asociación de Demografía Histórica; 1998; Muñoz Pradas, Francisco. Pautas territoriales de mortalidad en la España de 1860: una reconstrucción y análisis. Revista de Demografía Histórica. 2005; 23 (2): 43-78; Pujadas-Mora, Joana-Maria. L'evolució de la mortalitat infantil i juvenil a la ciutat de Palma (Mallorca, 1838-1960). Palma: Universitat de les Illes Balears; 2009. 
duplicación de los efectivos de una determinada población sin que el ritmo de crecimiento variase a lo largo del tiempo. Junto a estos cálculos se estimó la densidad de la población para mostrar el grado de saturación del espacio en relación a los recursos económicos. Ambos cálculos constituyeron las bases para las proyecciones de población del momento ${ }^{30}$, aunque cuando Fajarnés utilizó estas medidas para las Baleares no aventuró ninguna cifra de población para el futuro.

Fajarnés tituló sus estudios sobre el crecimiento de la población con el apelativo de Acrecentamiento $^{31}$. En una primera fase atendió al crecimiento de la población balear por años censales desde 1857 hasta 1887. Posteriormente utilizó censos de época preestadística como el de Floridablanca (1787) o el de Godoy (1797), estimaciones realizadas en diccionarios geográficos como los de Miñano y Madoz en 1826 y 1847-1848 respectivamente, o toda una serie de revisiones padronales de mediados del siglo XIX. La cuantificación del crecimiento el autor también la incorporó como introducción en otros trabajos en que se trataba la dinámica demográfica.

En el estudio de la naturaleza del crecimiento de la población urbana, y más en concreto, el de la capital de la provincia - la ciudad de Palma-, incorporó la distribución de sexos como otra medida agregada del estado de salud. Es decir el desequilibrio de sexos existente en la ciudad de Palma a finales del siglo XIX se debía, en parte, a la mayor mortalidad del sexo masculino. Aunque también se explicaba por el mayor nacimiento de niñas, la emigración en solitario de los hombres y la mayor proporción de religiosas y de personal doméstico femenino que no se veía compensada por las guarniciones militares existentes en la capital ${ }^{32}$. Completó estas conclusiones con la comparación con el resto de capitales y provincias españolas y con algunos países europeos ${ }^{33}$.

30. Otero, Hernán. Sueños cifrados. Una arqueología de las proyecciones de población en la Argentina moderna. Revista de Demografía Histórica. 2004; 22 (1): 209-239.

31. La obra más representativa de este tipo de estudios es: Fajarnés Tur, n. 24.

32. Fajarnés Tur, Enric. Distribución de sexos en las Islas Baleares. Palma: Tipografía de las hijas de Juan Colomar; 1903.

33. Fajarnés Tur, Enric. Demografía retrospectiva de la isla de Menorca. Los pequeños núcleos de población en Menorca. Revista Balear de Ciencias Médicas. 1902; 22: 49-57; 106-109. 


\subsection{La estacionalidad de la mortalidad en la búsqueda de la relación entre clima y salud}

La tradición ambientalista hipocrática seguía viva a mediados del siglo XIX, como mostró Fernando Weyler ${ }^{34}$ en su Topografía físico-médica de las islas Baleares y en particular de Mallorca (1854); especialmente en el capítulo titulado Meteorología:

«La creencia de que estamos sometidos al influjo del aire, estaciones, clima, y en una palabra, al de todos los fenómenos, cuyo conjunto forma la ciencia meteorológica, es tan antigua como los primeros escritos de los hombres, y léjos (sic) de debilitarse con el transcurso de los tiempos, por el contrario toma mayores creces, á (sic) medida que se van estudiando los paises (sic) y los meteoros, y por consiguiente las causas de las enfermedades.» ${ }^{35}$.

El médico militar Weyler explicaba que en las Baleares durante el invierno dominaba la constitución catarral e inflamatoria; en primavera, la inflamatoria exclusivamente; en verano también la inflamatoria, la biliosa, la biliosa inflamatoria con degeneración al adinamismo, ataxismo, tifoidismo; y en otoño, la biliosa e intermitente. Estas constituciones médicas:

«se armonizan con el clima y situación topográfica; porque como pais (sic) templado, las inflamaciones en invierno no son tan puras é (sic) intensas, como en las regiones mas (sic) septentrionales; y las biliosas, pútridas, tifódicas, etc., no llegan al alta grado con que se presentan en parages (sic) mas (sic) calurosos» ${ }^{36}$.

De este modo, el clima templado, húmedo, variable, poco lluvioso y de escasas tempestades propio del país balear se presentaba más favorable para la restitución de la salud. Aunque en la topografía no se presentaran medidas propiamente cuantitativas, Weyler afirmaba que la mortalidad podía variar substancialmente entre un año y el siguiente y que cuando se

34. Sobre el médico militar Weyler se puede ver: Tejerina, José María. Don Fernando Weyler y Laviña, médico y escritor del siglo XIX. Medicina e Historia. 1972; 17: 7-26.

35. Weyler Laviña, Fernando. Topografía físico médica de las islas Baleares y en particular de la de Mallorca. Palma: El Tall; 1992 [Edición facsímil de 1854], p. 41.

36. Weyler Laviña, n. 35, p. 201. 
tienen en cuenta las estaciones se observan diferencias significativas en el número de defunciones:

«las diferencias son mas (sic) constantes y pueden formularse en el siguiente órden ( $\mathrm{sic}$ ): $1^{\circ}$ verano, $2^{\circ}$ invierno, $3^{\circ}$ otoño y $4^{\circ}$ primavera. Hasta en los meses pudiera marcarse una sucesion (sic), y fundar un calendario médico-necrológico, que aproximadamente es el siguiente: agosto, setiembre (sic), julio, diciembre, noviembre, enero, octubre, febrero, marzo, abril, junio y mayo» ${ }^{37}$.

El cálculo preciso de la interacción del clima con la salud de la población a través de la medida de la mortalidad por estaciones o periodos del año se abordó en los estudios de Fajarnés, como explicamos a continuación.

Fajarnés, hijo de la medicina de laboratorio, afirmaba que las estaciones en la mortalidad:

«obran de dos maneras, cuyos límites de acción no es posible todavía precisar: obran directamente sobre el organismo humano como agentes físicos, é indirectamente modificando las propiedades vitales de los gérmenes productores de los procesos infecciosos» ${ }^{38}$.

La cuantificación de la mortalidad por meses fue frecuente en los estudios demográficos e epidemiológicos de este médico. Normalmente este tipo de mediciones se acompañó con el estudio de la mortalidad diferencial por sexos o por edades. Aunque únicamente destacaremos uno de sus títulos dedicados en exclusiva a la cuestión, Influencia de las estaciones en la mortalidad de Palma de Mallorca, que fue publicado en forma de folleto en 1902.

En este contexto no puede dejar de mencionarse el discurso inaugural del año académico de 1891 de la Real Academia de Medicina y Cirugía de Palma, presentado por el médico Antoni Mayol Vidal bajo el título de Datos y observaciones acerca los caracteres médico-metereológicos del clima de Palma, en sus relaciones con el de Mallorca y con la climatología general ${ }^{39}$. En éste realizó una descripción detallada del clima de Palma, titulado «Elementos

\footnotetext{
37. Weyler Laviña, n. 35, p. 258.

38. Fajarnés Tur, Enric. Influencia de las estaciones en la mortalidad de Palma de Mallorca. Palma: Tipografía de las hijas de Juan Colomar; 1902, p. 6.

39. Ambas obras, la de Fajarnés y la de Mayol, tienen un claro parangón con la de los ingleses Guy y Cantab en su artículo An attempt to determine the influence of the season and weather on sickness and mortality publicado en 1843 en Journal of the Statistical Society of London
} 
atmosféricos del clima de Palma», donde presentó «la composición química de la atmósfera, la temperatura atmosférica, la movilidad atmosférica, la presión atmosférica y la luminosidad atmosférica». En la parte final de este discurso, de hecho la parte más relevante para nuestro estudio, destacó la influencia estacional en los nacimientos, concepciones y defunciones ${ }^{40}$.

Fajarnés (1902) utilizó los datos de defunciones estacionales ocurridas entre 1880 y 1894 y relativos a Palma, mientras Mayol (1891) se refería al período 1885-1890. Ningún de los dos autores apuntó el origen de sus datos, aunque posiblemente Mayol utilizara los estadillos mensuales conocidos como Demografía médica, que publicaba la Revista Balear de Ciencias Médicas desde su creación en 1885. Los datos de Fajarnés podrían ser de elaboración propia a partir de la información del Registro Civil de la ciudad de Palma, ya que fuentes de carácter general como el Movimiento natural de la población no ofrecieron tanto detalle para las defunciones.

En los análisis de ambos autores se relacionaron las estaciones con las causas de defunciones (Mayol utilizó una división entre enfermedades infecciosas, comunes y violentas, siguiendo la distribución de los estadillos mensuales) y con las edades a la defunción.

\subsection{El perfil demográfico y epidemiológico de la población}

La construcción del perfil demográfico y/o epidemiológico local a finales del siglo XIX permitió identificar las enfermedades llamadas evitables, dado el conocimiento de su etiología, que afectaban a la población y por ello justificar con estos datos la reforma sanitaria que tan largamente clamó el Higienismo.

Eusebi Estada ${ }^{41}$ en su obra La Ciudad de Palma: su industria, sus fortificaciones, sus condiciones sanitarias y su ensanche, con un apéndice

como menciona Rau, Ronald. Seasonality in human mortality: a demographic approach. Rostock: University of Rostock; 2005.

40. Mayol Vidal, Antoni. Datos y observaciones acerca de los caracteres médico-meteorológicos del clima de Palma en sus relaciones con el de Mallorca y con la climatología general (discurso inaugural leído en la sesión pública celebrada por la Real Academia de Medicina y Cirugía de Palma de Mallorca el 25 enero de 1891). Palme de Mallora; s.e; 1891, p. 330.

41. Para una aproximación a la figura de Eusebi Estada véase el estudio introductorio realizado por Francesc Bujosa Homar y Miquel Seguí Aznar en la edición facsímil de Estada Sureda, Eusebi. La Ciudad de Palma: su industria, sus fortificaciones, sus condiciones sanitarias y 
sobre las condiciones que han de reunir las viviendas para ser salubres (1885 y reeditada y aumentada en 1892) utilizó el hecho demográfico para justificar la necesidad del derrumbe de las murallas de la ciudad de Palma ${ }^{42}$. Afirmó que la mortalidad de la provincia balear era elevada como consecuencia de la influencia de la ciudad de Palma, su capital. Una ciudad que no crecía demográficamente por su propia dinámica sino por la atracción migratoria que ejercía sobre el resto de municipios de la isla, eminentemente rurales. Aunque las cifras de mortalidad de la ciudad de Palma indicaban que:

«es una de las capitales de provincia de menor mortalidad, puesto que no excede (sic) anualmente de 24 á (sic) 25 por mil. Esto es cierto, y no hay motivo sino para felicitarse por ello, pero también lo es, en mi concepto, que este resultado no es debido, en manera alguna, á sus buenas condiciones sanitarias, sino á (sic) la bondad de nuestro clima, á (sic) la moderación de nuestras costumbres, á (sic) nuestra vida sosegada y tranquila - aparte de que esta mortalidad que es pequeña en España, es grande comparada con la de otros países - y el estar contenida dentro de límites relativamente bajos no significa nada en absoluto, no significa otra cosa sino que hay quién está peor que nosotros, sin que, en manera alguna, arguya que no pudiera mejorarse reformando sus actuales condiciones sanitarias» ${ }^{43}$.

Una cuestión demográfica que objetivamente no le servía para justificar la pretendida situación sanitaria deplorable, por lo que optó por comparar la ciudad de Palma con realidades europeas con evidentes recorridos histórico-demográficos diferentes, con transiciones demográficas más avanzadas que la española y que la propia balear, pese a la precocidad de

su ensanche, con un apéndice sobre las condiciones que han de reunir las viviendas para ser salubres. Palma: Tipografía de Viuda e Hijos de Pedro J. Gelabert; 1885, publicada por la Conselleria d'Innovació i Energia en 2003 en su colección «La Ciència a les Illes Balears» y March Noguera, Joan. L'enderrocament de les muralles de Palma. Un triomf de l'higienisme mallorquí. Gimbernat. 2002; 37: 247-263.

42. El derrumbe definitivo de las murallas de la ciudad de Palma no se llevó a cabo hasta 1902. No obstante, fue un elemento recurrente en el discurso de denuncia médico principalmente del último tercio del siglo XIX como se explica en el artículo en prensa de la autora titulado «Reforma sanitaria y movimiento puericultor en la lucha contra la mortalidad infantil en la ciudad de Palma de Mallorca (siglos XIX-XX)». Una aproximación bibliográfica local puede encontrarse en el monográfico: Les murades de Palma. Estudis baleàrics. 2002; 70-71, con motivo del centenario de su derrumbe.

43. Estada Sureda, n. 41, p. 10. 
ésta. Comparaciones que realizó a partir de tasas brutas de mortalidad utilizando indistintamente provincias, regiones o países.

Aparte de estudios que recogen sólo el perfil demográfico, como el que hemos presentado se escribieron trabajos de corte epidemiológico. Por una parte destacaremos los trabajos en los que se utilizaron determinadas enfermedades como la fiebre tifoidea para ejemplificar la necesidad de modernización sanitaria. Citaremos dos estudios en que su justificación discursiva partía de la utilización de las cifras de mortalidad de la expresada causa. El primero de éstos, Enfermedades infecciosas en la ciudad de Palma durante el invierno de 1899, era un informe encargado por el Ayuntamiento de la ciudad de Palma a la Real Academia de Medicina y Cirugía de la misma ciudad, con el propósito de dirimir las enfermedades reinantes en la capital.

En este informe se utilizó la mencionada enfermedad como punta de lanza para la justificación de la necesidad de una actuación sanitaria en la ciudad, aunque la cuantificación del informe no se refirió exclusivamente a esta enfermedad. Se mostró el porcentaje de defunciones por causa infectocontagiosa sobre el total de defunciones ocurridas entre 1880 y 1894 con la pretensión de mostrar que esta mortalidad podía ser evitable. A su vez reveló que las enfermedades prevalentes del momento eran la gripe, la angina lardácea y la fiebre tifoidea sin ninguna estimación de número de afectados o de defunciones, posiblemente porque estas conclusiones venían esbozadas a partir de los estados de salud quinquenales debatidos en las sesiones de la Real Academia de Medicina y Cirugía. El formato del estudio, posiblemente por su inmediatez al hecho concreto, imposibilitó dar cifras y ofrecer cálculos más profundos. Aun así Fajarnés, autor del informe, afirmaba que la fiebre tifoidea no revestía gravedad, aunque se había convertido en una enfermedad endémica.

La estimación de la mortalidad por esta causa específica también le sirvió al médico Bernat Riera Alemany como justificación de la necesidad del Saneamiento de Palma, título del ciclo de cinco conferencias que impartió. Según este médico, entre 1884 y 1893 se habían producido en la ciudad de Palma 341 defunciones por esta causa. Con este número de defunciones estimó una media anual de 34 muertes al año, aunque en términos de morbilidad explicaba que cada año 500 personas enfermaban de fiebre tifoidea. Si el número de muertos y de enfermos se traducía en número de días de trabajo perdidos, éste ascendía a 12.500 dietas. A esta tormenta de cifras le incorporó la comparación de la tasa bruta de defunciones por 
esta causa de la ciudad de Palma con la de Viena, 5,63 por cada diez mil habitantes frente al $1,30^{44}$.

Junto a estos cálculos presentó las cifras globales de mortalidad de la ciudad de Palma alegando que debían ser iguales a las de las ciudades inglesas ( sin especificar cuáles) dado que la capital balear tenía unas circunstancias climatológicas más favorables. Comparaba la tasa del 19,08 por mil de las ciudades inglesas, con el 23,70 por mil de la ciudad de Palma, lo que implicaba - según el autor- una diferencia absoluta de 300 defunciones. En este caso no cifró las pérdidas económicas por la no percepción de salarios sino que a cada vida perdida se le asignó un valor monetario. Esta cuantificación servía para ejemplificar que una intervención sanitaria pública sería menos costosa que el valor de estas vidas perdidas.

Con carácter más general el estudio titulado El nostre estat sanitari del médico Emili Darder Cànaves publicado en 1928 en la revista La Nostra Terra, también argumentó la benevolencia de las cifras de mortalidad de las Baleares. Corresponde a un trabajo entre la investigación y la divulgación científica de una línea de investigación que había iniciado el autor a principios de la década de los años 20 bajo el título general de «Lucha contra las enfermedades evitables». El estudio concreto muestra el contexto sanitario por medio de la comparación de las tasas brutas de mortalidad por causas de defunción de la provincia balear y de su capital, con el resto de provincias y capitales españolas y con un grupo de «naciones y capitales avanzadas» como Nueva Zelanda, Dinamarca, Estados Unidos, Inglaterra, Gales, Escocia, Holanda, etc. Organizó sus conclusiones por tipo de causas de defunción. Con relación a las enfermedades pestilenciales (peste, cólera y fiebre amarilla) afirmaba que «hace tiempo que estamos libres de las incursiones de las tres pestes exóticas capitales» ${ }^{45}$. Además «el tifus exantemático y el tifus recurrente - enfermedades de la suciedad y de la miseria - no están registradas en la historia médica de Mallorca» ${ }^{46}$.

El bajo número de muertes por causa de tos ferina, difteria y escarlatina situaban a las Islas Baleares en la mejor de las posiciones, a nivel nacional

44. Riera Alemany, Bernat. El saneamiento de Palma. Revista Balear de Ciencias Médicas. 1902, 23: 1-14, 25-36, 49-63, 73-84.

45. Darder Cànaves, Emili. El nostre estat sanitari. Palma: Imprenta de Francisco Soler; 1928, p. 2. El texto originalmente fue escrito en catalán. La traducción se debe a la autora del artículo.

46. Darder Cànaves, n. 45, p. 2. 
e incluso mundial ${ }^{47}$. No obstante, las muertes causadas por sarampión y gripe colocaban a la provincia de las Baleares en la zona media del ranking sanitario español. A su vez, concluía que la mortalidad por tuberculosis en la ciudad de Palma era de poca intensidad en comparación a la del resto de capitales. El paludismo, afirmaba el autor, había dejado de ser una causa primordial de mortalidad como consecuencia de la desecación de muchas zonas pantanosas en Mallorca.

$\mathrm{Su}$ conclusión era que las Islas Baleares, en términos demográficos, y refiriéndose a la mortalidad «iban regularmente por encima de España, lo cual representa nuestra mayor victoria» ${ }^{48}$ debido a la benignidad del clima y a «una mayor limpieza y más buena posición económica general de nuestros insulares ${ }^{49}$. Aun así, este discurso se volvía derrotista cuando la mortalidad balear se comparaba con las ciudades cultas europeas dadas sus diferencias en el clima y su recorrido histórico:

«Si vivimos con estas condiciones favorables, es perdonable que estemos fuertemente atrasados en comparación de los países cultos, de clima mucho más malo que el nuestro y que muchos de ellos se tuvieron que rehacer después de la guerra?» ${ }^{50}$.

El estado de la salud de la población balear no solo fue materia de revisiones dispersas en el tiempo, como hemos presentado con la producción de los anteriores autores, sino que de manera periódica la Real Academia de Medicina y Cirugía de Palma debatió sobre este tema tanto en juntas ordinarias como extraordinarias ${ }^{51}$.

El apartado de «Enfermedades reinantes» corresponde al informe sobre el estado general de la salud de la población a partir de un resumen de la morbilidad y mortalidad casi exclusivamente de la ciudad de Palma y solo se mencionan otras localidades cuando se vieron afectadas por alguna epidemia. Estos estados eran de carácter retrospectivo y se referían a los 15 días anteriores a la junta, es decir, el periodo ordinario transcurrido entre

\footnotetext{
47. Darder Cànaves, n. 45, p. 2.

48. Darder Cànaves, n. 45, p. 4.

49. Darder Cànaves, n. 45, p. 4.

50. Darder Cànaves, n. 45, p. 7.

51. Artículo 10 del capítulo IV: De las Juntas académicas del Real Decreto de 28 de agosto de 1830 sobre «El reglamento general para el régimen literario é interior de las Reales Academias de Medicina y Cirugía del Reino».
} 
juntas. Con frecuencia eran informes de corta extensión dado que se trataba de un resumen realizado por el secretario de la academia después del debate de los miembros de la junta sobre las enfermedades que ellos mismos habían diagnosticado y tratado durante este periodo. En el periodo estival su extensión se ampliaba ya que abarcaban una cronología más amplia.

Los estados de salud presentados por los médicos Fajarnés, Riera y Darder y por el ingeniero Estada y los propios de la Real Academia de Medicina parten de dos consideraciones diferentes que convergen en una única finalidad, la lucha contra la mortalidad. Los primeros utilizaron este tipo de artículos para crear un estado de opinión sobre la salud de la población, mientras que la Real Academia de Medicina tenía otras finalidades, como el velar por la salud pública, estimular la enseñanza y el progreso de la medicina, igual que proporcionar auxilio científico a las juntas de sanidad locales, tanto municipales como provinciales ${ }^{52}$. Precisamente en el marco de estas finalidades hay que entender los resúmenes sanitarios de menor calado estadístico y de mayor carácter clínico, presentados anteriormente ${ }^{53}$.

\section{Valoraciones finales}

Los higienistas baleares se sirvieron de los cálculos estadísticos para mostrar la necesidad de una reforma sanitaria con el fin de modernizar la sociedad. Con el tiempo se ha demostrado que sus métodos estadísticos no fueron del todo correctos y sus comparaciones fueron bastante asimétricas. Por ello, pese al tono discursivo de denuncia de los textos higienistas presentados, se tuvo que reconocer el buen estado de salud de la población balear; el cual, para los facultativos contemporáneos, se debía casi exclusivamente

52. Artículo único del capítulo IX: Salud Pública del Real Decreto n. 57.

53. Ciertamente los apartados dedicados a las enfermedades reinantes podría decirse que adolecieron de falta de estimaciones numéricas. No obstante suponemos que sería erróneo pensar que la Real Academia de Medicina de Palma mantuviese la misma posición que su homóloga francesa ante las investigaciones estadísticas, que reputaba de inútiles para la clínica. Sobre el tema ver Schweber, n. 12. Distinguidos miembros de la academia insular cultivaron este tipo de investigaciones largamente, destacando entre ellos Enric Fajarnés. Un debate que también se presentó a escala nacional en el Congreso Médico de 1888 tras las aportaciones de carácter estadístico del médico barcelonés Josep Nin. Se recomienda ver Rodríguez Ocaña, n. 16. 
a la benevolencia del clima insular por la cual era necesaria y quedaba justificada la reforma sanitaria.

En estos cálculos se plasmó el crecimiento de la población, el perfil demográfico y epidemiológico de la mortalidad y su relación con el clima. No se establecieron conexiones entre la mortalidad y las diferencias socioeconómicas a través del uso de la clase social ni tampoco se atendió a la cuantificación de la mortalidad infantil como sí se realizó en Barcelona, Madrid o Zaragoza ${ }^{54}$. Cierto es que en los escritos tratados tampoco se refiere a la tuberculosis, la enfermedad social por excelencia, existiendo toda una producción, aunque no muy extensa, a partir de la segunda década del siglo XX.

El médico Fajarnés Tur, aunque casi nunca ejerció la medicina, ha sido citado constantemente a lo largo de este trabajo por su gran afán cuantificador. En el contexto balear debe ser considerado como el introductor del método estadístico para el análisis demográfico y epidemiológico, en parangón con los médicos barceloneses Josep Nin y Lluis Comenge ${ }^{55}$. Seguramente en otras capitales y más si se tienen en cuenta las instituciones sanitarias como institutos de higiene, las reales academias, los colegios médicos o la misma Sociedad Española de Higiene los ejemplos individuales de este tipo de perfil investigador proliferarían. Sería interesante contar con estudios similares con el fin de generar una visión más completa de la cuantificación en Salud Pública española.

\section{Agradecimientos}

Me gustaría agradecer a mi directora de tesis, la Dra. Isabel Moll sus comentarios y a los revisores de Dynamis sus sugerencias, que han ayudado a mejorar este artículo.

54. Perdiguero Gil, Enrique; Robles González, Elena. La protección a la infancia y la Sociedad Española de Higiene. En Perdiguero Gil, Enrique, comps. Salvad al niño. Estudios sobre la protección a la infancia en la Europa mediterránea a comienzos del siglo XX. Valencia: Seminari d'Estudis sobre la Ciencia; 2004, p. 93-120; Rodríguez Ocaña, n. 16; Rodríguez Ocaña, Esteban; Perdiguero Gil, Enrique. Ciencia y persuasión social en la medicalización de la infancia en España, siglos XIX-XX. Historia, ciencias, saúde-Manguinhos. 2006; 13 (2):1-20.

55. Rodríguez Ocaña, n. 16. 


\section{Demographic and epidemiological quantification in Balearic hygienism, 1850-1930}

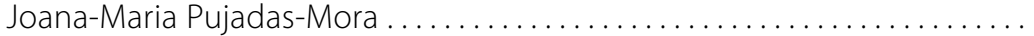

1.-Introduction. 2.-Quantification efforts and methods. 3.-Elements configuring the health status of the population. 3.1.-The growth and gender distribution of the population. 3.2.-The seasonality of mortality in the search for a relationship between climate and health. 3.3.-The demographic and epidemiologic profile of the population. 4.-Final evaluations.

ABSTRACT: At the end of the 19th century, social medicine promoted the use of quantification as a means to evaluate the health status of populations. In Majorca, hygienists such as the physicians Enric Fajarnés, Bernat Riera, Antoni Mayol and Emili Darder and the civil engineer Eusebi Estada sought a better understanding of health status by considering the population growth, the demographic and epidemiological profile and the influence of weather on mortality. These calculations showed that the Balearic population had a good health status in comparison to the population of mainland Spain, although less so in the international context. These results were explained by the benevolence of the insular climate, a factor that would also guarantee the success of the public health reforms proposed. 